\title{
Cluster-cluster aggregation of Ising dipolar particles under thermal noise
}

\author{
Masaru Suzuki, ${ }^{1, *}$ Ferenc Kun, ${ }^{2, \dagger}$ and Nobuyasu Ito ${ }^{3, \$}$ \\ ${ }^{1}$ Department of Applied Quantum Physics and Nuclear Engineering, Kyushu University, \\ 744 Motooka, Nishi-ku, Fukuoka 819-0395, Japan \\ ${ }^{2}$ Department of Theoretical Physics, University of Debrecen, P.O. Box 5, H-4010 Debrecen, Hungary \\ ${ }^{3}$ Department of Applied Physics, The University of Tokyo, 7-3-1, Hongo, Bunkyo-ku, Tokyo 113-8656, Japan
}

(Received 3 January 2009; published 14 August 2009)

\begin{abstract}
The cluster-cluster aggregation processes of Ising dipolar particles under thermal noise are investigated in the dilute condition. As the temperature increases, changes in the typical structures of clusters are observed from chainlike $(D \simeq 1)$ to crystalline $(D \simeq 2)$ through fractal structures $(D \simeq 1.45)$, where $D$ is the fractal dimension. By calculating the bending energy of the chainlike structure, it is found that the transition temperature is associated with the energy gap between the chainlike and crystalline configurations. The aggregation dynamics changes from being dominated by attraction to diffusion involving changes in the dynamic exponent $z=0.2$ to 0.5 . In the region of temperature where the fractal clusters grow, different growth rates are observed between charged and neutral clusters. Using the Smoluchowski equation with a twofold kernel, this heteroaggregation process is found to result from two types of dynamics: the diffusive motion of neutral clusters and the weak attractive motion between charged clusters. The fact that changes in structures and dynamics take place at the same time suggests that transitions in the structure of clusters involve marked changes in the dynamics of the aggregation processes.
\end{abstract}

DOI: 10.1103/PhysRevE.80.021402

PACS number(s): 61.43.Hv, 45.50.-j, 83.10.Pp

\section{INTRODUCTION}

The patterns and dynamics of cluster-cluster aggregation (CCA) have been studied both experimentally and theoretically [1-7]. The CCA processes of dipolar particle systems have been attracting particular attention in recent years [8-11], because they exhibit various aspects of CCA due to the long-range and/or anisotropic interaction.

There have been attempts to classify various CCA processes using physical values such as the fractal dimension of the cluster structure or the dynamic exponent of the cluster evolution $[1,2]$. In many CCA processes, the time evolution of the average cluster size $\bar{s}(t)$ is known to exhibit power-law behavior: $\bar{s}(t) \sim t^{z}[3,4]$. In the case of diffusion-limited CCA (DLCA) where particles only experience short-range interactions and each cluster moves diffusively with a cluster diffusion constant proportional to $s^{\gamma}$ ( $s$ : cluster size, the value of the exponent $\gamma$ is negative for normal diffusion), the dynamic exponent $z$ is analytically described as

$$
z=\frac{1}{1-\gamma+(2-d) D},
$$

where $d$ is the spatial dimension and $D$ is the fractal dimension of the clusters [5]. On the other hand, in the attractionlimited CCA (ALCA), where clusters attract each other through long-range interaction and thermal diffusion is negligible, the dynamic exponent is

\footnotetext{
*suzuki@athena.ap.kyushu-u.ac.jp

†feri@dtp.atomki.hu

†ito@ap.t.u-tokyo.ac.jp
}

$$
z=\frac{1}{1+(\alpha+1) / d}
$$

when the attractive force between clusters is proportional to $r^{-\alpha}$ with intercluster distance $r$ [8].

CCA in oppositely charged particles such as electrically charged particles or Ising dipoles (introduced in the following paragraph) is more complicated, because of the frustration of the particle charges $[6,7,12]$. In such systems, clusters containing even and odd numbers of particles evolve differently over time, since the total charge of a even-size cluster is neutral while an odd-size cluster has a nonzero total charge. This phenomenon is called hetero aggregation. The dynamic exponent may also differ from that of monodisperse systems.

Recently, the CCA process of a binary monolayer of Ising dipolar particles (IDPs) has been intensively investigated [12-15]. IDPs are numerical model to simulate some experimental systems of dipolar particles [16,17], in which the dipole orientation of each particle is fixed to upward or downward perpendicular to the plane of motion. The interaction of particles is isotropic and particles can be regarded as oppositely charged particles. The structures and dynamics of IDPs in the zero-temperature environment have been investigated. Various types of structures are observed for IDP systems, and the structures are classified on the basis of the relative dipole moment and relative size of particles $[13,14]$. Yoshioka et al. [12] investigated the ALCA dynamics of IDP systems. They estimated the dynamic exponent to be $z \simeq 0.2$, which is much smaller than that predicted by Eq. (2), due to the hetero dynamics of IDP. The origin of the value $z=0.2$ has not yet been explained theoretically.

In the present article, the structures and dynamics of CCA of IDPs under small thermal noise is investigated. Structures observed under the zero-temperature environment are rather 
fragile [14], i.e., only a small energy barrier must be overcome to change their shapes. The structures of the clusters, therefore, may change under finite thermal noise. Such changes in structures are thought to also involve changes in their dynamics. Furthermore, investigation of CCA at finite temperatures is expected to more vividly illustrate the mechanism of aggregation in this system. The heteroaggregation process should be well controlled by temperature, since the attractive motion is weakened relative to the diffusive motion as the temperature increases.

The present article is organized as follows: the model and method are described in Sec. II. The numerical results are given in Sec. III and the analytical analyses are presented in Sec. IV. Sections V and VI are devoted to a summary and a discussion, respectively.

\section{MODEL AND METHOD}

The system consists of $N$ IDPs in a square box of side $L$ with a periodic boundary condition. All the particles have identical mass. Each particle has a spherical elastic volume of radius $R$ and a pointlike dipole moment at the center. The dipole moment of each particle is fixed throughout the time evolution, is perpendicular to the plane of motion and points either upward or downward. The pair potential between the $i$ th and the $j$ th particles is

$$
\Phi\left(r_{i j}\right)=\Phi^{\mathrm{pp}}\left(r_{i j}\right)+\frac{\mu_{i} \mu_{j}}{r_{i j}^{3}},
$$

with the dipole moments $\mu_{i}$ and $\mu_{j}$ of the $i$ th and the $j$ th particles, respectively, and the interparticle distance $r_{i j}$. The strength of each dipole moment is either with $\mu_{1}\left(\equiv \mu_{0}\right)$ or $\mu_{2}\left(\equiv-\mu_{0}\right)$. The relative number of particles with moment $\mu_{1}$ to that with moment $\mu_{2}$ is unity. The core potential is denoted by $\Phi^{\mathrm{pp}}$. To improve the efficiency of the particle dynamics simulation, a slight overlap of particles is allowed and the interaction is assumed to satisfy the Hertzian contact potential

$$
\Phi^{\mathrm{pp}}\left(r_{i j}\right)= \begin{cases}\frac{2}{5} k_{\mathrm{pp}}\left(2 R-r_{i j}\right)^{\frac{5}{2}} & \left(r_{i j} \leq 2 R\right), \\ 0 & \left(r_{i j}>2 R\right),\end{cases}
$$

with the elasticity constant $k_{\mathrm{pp}}=25 \mu_{0}^{2} /(2 R)^{11 / 2}$.

We perform a series of Langevin dynamics simulations. The difference equation used to update a particle from time $t$ to $t+\Delta t$ is

$$
\boldsymbol{v}(t+\Delta t)=e^{-\zeta \Delta t} \boldsymbol{v}(t)+\frac{1}{m \zeta}\left(1-e^{-\zeta \Delta t}\right) \frac{d \Phi}{d \boldsymbol{r}}+\delta \boldsymbol{v}^{\mathrm{B}},
$$

with the particle mass $m$ and the friction coefficient $\zeta, \delta \boldsymbol{v}^{\mathrm{B}}$ $\equiv\left(\delta v_{x}^{\mathrm{B}}, \delta v_{y}^{\mathrm{B}}\right)$ is a random vector sampled from the GAUSSIAN distribution

$$
\exp \left[\left(\delta v_{x}^{\mathrm{B}^{2}}+\delta v_{y}^{\mathrm{B}^{2}}\right) /\left(2 \sigma^{2}\right)\right]
$$

with the variance
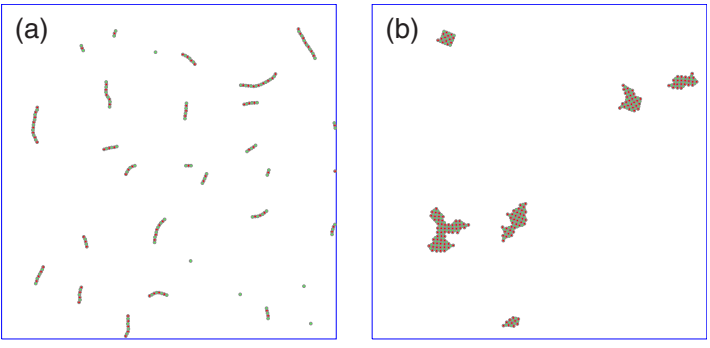

FIG. 1. (Color online) Typical snapshots of cluster-cluster aggregation in IDP systems at (a) $T=0.001$ and (b) $T=0.02$. Small parts of the system are shown for the clarity, which size is $250 R$ $\times 250 R$ for each. One can see the chainlike structure for the system at low temperatures, while the particles form a more compact structure at high temperatures.

$$
\sigma^{2}=\frac{2 \zeta k_{\mathrm{B}} T \Delta t}{m}
$$

We use unity for the values of $m$ and $\zeta$. We use the coverage density $\phi$, which is defined as $\phi \equiv N \pi R^{2} / L^{2}$. The reduced (dimensionless) temperature $T^{*}$ is given by

$$
T^{*}=\frac{k_{\mathrm{B}}}{2\left|\mu_{0}\right|^{2}(2 R)^{-3}} T,
$$

with the binding energy of two particles. Hereafter we use $T$ as the reduced temperature for the simplicity. In the following sections, the unit of time is set to be $\sqrt{4 m R^{5} / \mu_{0}^{2}}$ and the reduced time $t / \sqrt{4 m R^{5} / \mu_{0}^{2}}$ is denoted as $t$ for simplicity. We set the cut-off length of the dipolar interaction $R_{\mathrm{cf}}$ depending on the density to satisfy $R_{\mathrm{cf}}>(10 / \sqrt{\phi}) R$, in order that the interaction range is much longer than the distance between neighboring clusters at any stage of time evolution. Most of the simulations are performed using $N=6400$ particles.

\section{RESULTS}

The time evolution is observed for different values of temperature and density. The initial configuration is set to a uniform random distribution for each run. We study the range of temperature from $T=0.002$ to $T=0.04$ and density from $\phi=0.005$ to $\phi=0.2$. Because we are interested in the region where particles can aggregate into solid clusters, the highest temperature considered $(T=0.04)$ is below the solidification point $\left(T_{s}=0.059[18]\right)$. We observed the system within the time range where the sizes of growing clusters are much smaller than the system size so that the structure and dynamics are free from the finite-size effect.

Figure 1 shows typical configurations of aggregating clusters at different temperatures. While chainlike structures are dominant at low temperatures, compact structures appear at high temperatures. The compact structures contain some microcrystalline structures with square lattice order. We find that the size of the crystalline domain $(2 \times 2$ square, $3 \times 3$ square, $\cdots)$ tends to increase as the temperature is increased.

To characterize the structures of these clusters, their gyration radii are measured by sampling during the aggregation process. Here a cluster is defined as follows: two particles 


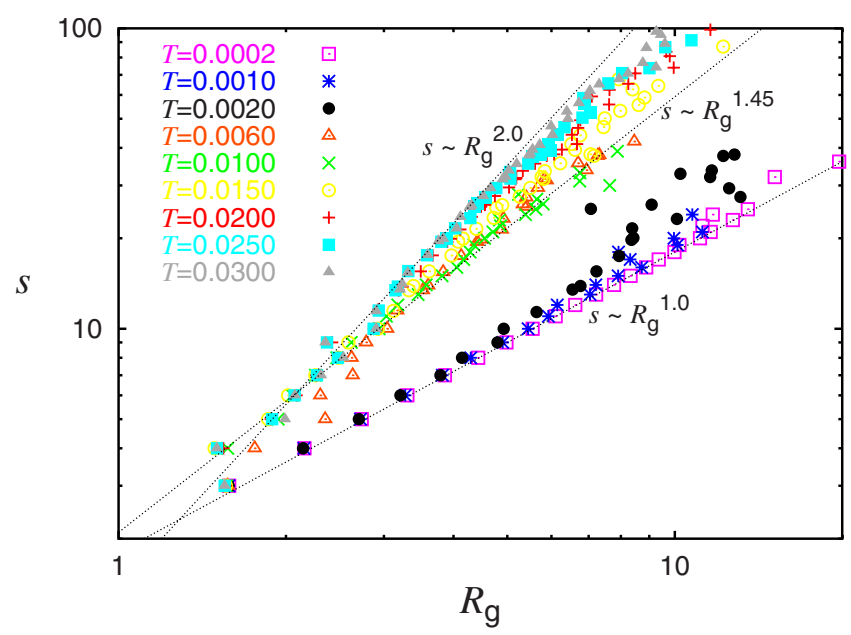

FIG. 2. (Color online) The gyration radius $R_{\mathrm{g}}$ versus cluster size $s$ for the system with $\phi=0.01$. As the temperature increases, three types of structures appear. The dotted lines denote the relationship $s \propto R_{\mathrm{g}}^{D}$ with $D=1, D=1.45$, and $D=2$. Decimal logarithms are taken for both axes.

are defined to be linked if the distance of them is less than $2.2 R$. A cluster consists of linked particles. The gyration radius $R_{\mathrm{g}}$ of a cluster with size $s$ is defined as

$$
R_{\mathrm{g}}^{2}(s)=\frac{1}{s(s-1)} \sum_{i<j}^{s} r_{i j}^{2},
$$

where the number of particles in the cluster is denoted by $s$. Figure 2 shows the gyration radius $R_{\mathrm{g}}$ at different temperatures plotted against the cluster size $s$. The fractal dimension $D$ is estimated by fitting data with the line denoting the relationship $s \propto R_{\mathrm{g}}^{D}$. The structure of an aggregate markedly changes between $T=0.002$ and $T=0.006$. The fractal dimension $D$ is approximately 1.0 when $T \leq 0.002$, reflecting the chainlike alignment of clusters. Above this temperature, the profile of $R_{\mathrm{g}}$ exhibits a crossover from $D \simeq 2.0$ at a relatively small cluster size to $D \simeq 1.45$ at a large cluster size. The region with $D=2.0$ corresponds to the existence of square microcrystalline domains. The crossover size $s_{\mathrm{c}}$ from $D$ $=2.0$ to $D=1.45$ at each temperature is estimated. As shown in Fig. 3, the crossover size tends to diverge as $s_{\mathrm{c}}(T)$ $\sim\left(T_{\text {crystal }}-T\right)^{-3.0}$ when $T_{\text {crystal }}$ is set to 0.04 . Thus, above $T$ $=0.04$, all the clusters are thought to have a normal square lattice structure. This crossover phenomenon occurring with changes in the fractal dimension of the cluster is discussed in the following section.

To investigate the dynamic properties of IDP aggregation, the time evolution of the average cluster size is observed. The average cluster size $\bar{s}$ is defined as

$$
\bar{s}=\frac{1}{N} \sum_{s} s^{2} n(s),
$$

where $n(s)$ is the number of clusters with size $s$. Figure 4 shows the time evolution of $\bar{s}$. For each curve, power-law growth, $\bar{s}(t) \sim t^{z}$ with the dynamic exponent $z$, appears as the

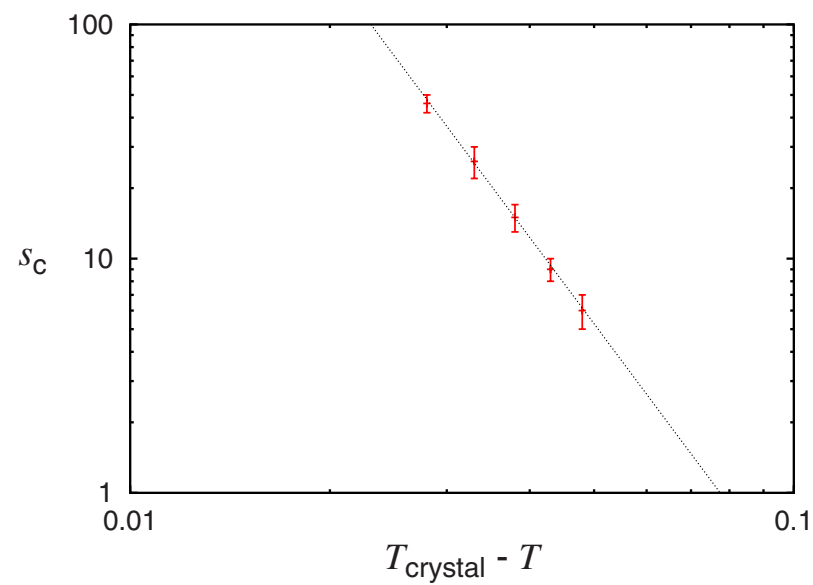

FIG. 3. (Color online) The temperature dependence of the crossover size $s_{\mathrm{c}}$. The cross over size from $D \simeq 2.0$ to $D \simeq 1.45$ was estimated. The horizontal axis is $T_{\text {crystal }}-T$, with $T_{\text {crystal }}=0.04$. Decimal logarithms are taken for both axes. The profile of $s_{\mathrm{c}}$ is well fitted by the dotted line denoting the relationship $s_{\mathrm{c}} \propto\left(T_{\text {crystal }}\right.$ $-T)^{-3.0}$.

asymptotic behavior. The estimated values of $z$ for different temperatures and densities are shown in Fig. 5. Although the dynamic exponent is known to converge to $z \simeq 0.2$ at $T=0$ in the dilute limit $\phi \rightarrow 0$ (estimated by Yoshioka et al. in [12]),
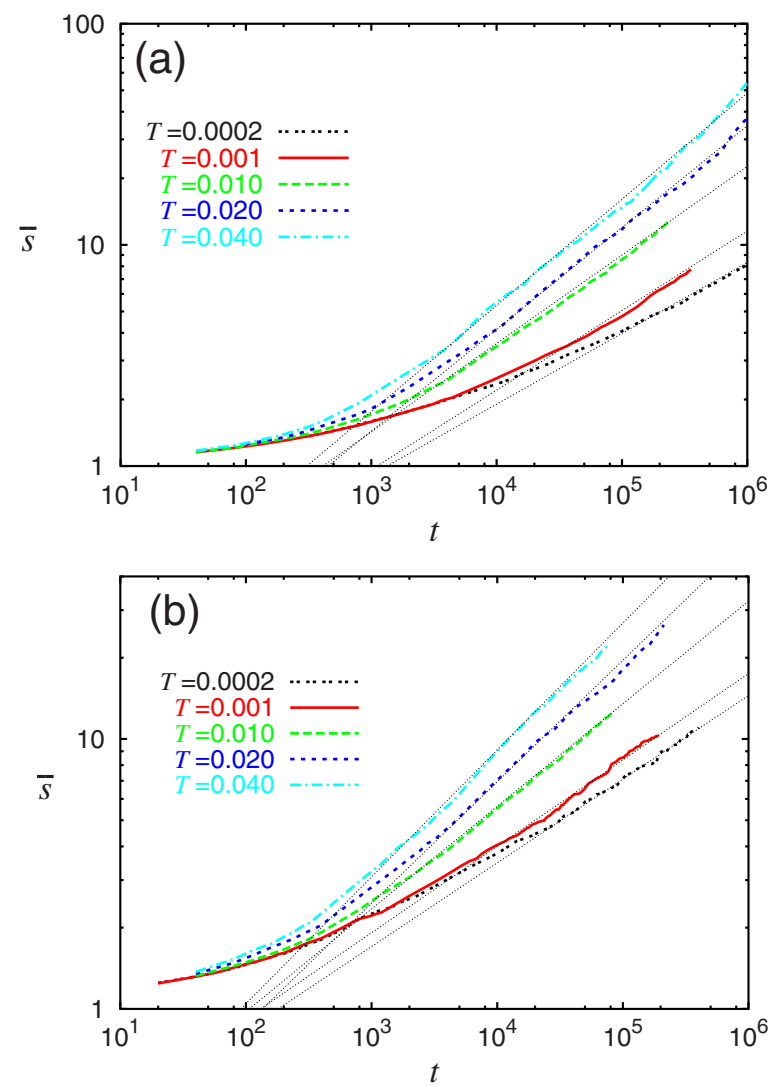

FIG. 4. (Color online) The time evolution of average cluster size for (a) $\phi=0.010$ and (b) $\phi=0.020$. Decimal logarithms are taken for both axes. The asymptotic behavior of the cluster size obeys a power law, as fitted by the dotted lines, and the values of the dynamic exponent $z$ are obtained from their slope. 


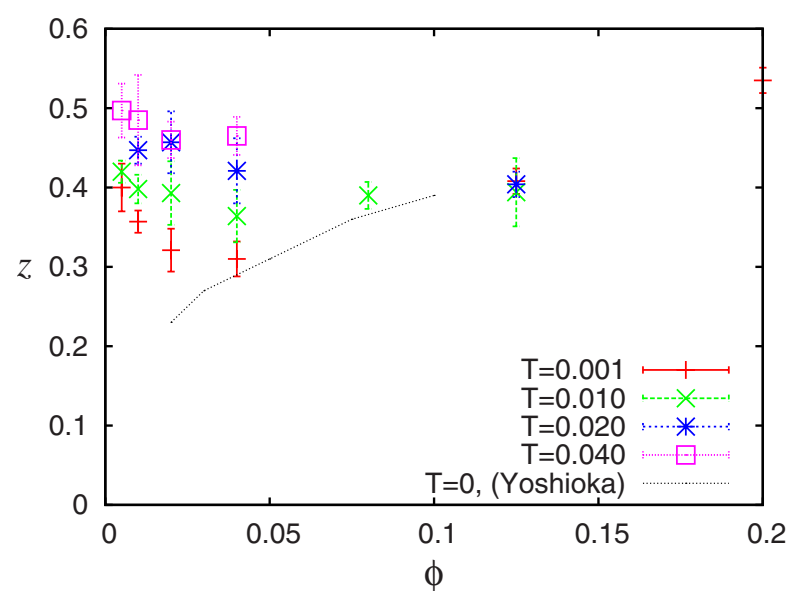

FIG. 5. (Color online) The dynamic exponent $z$ for each temperature and density. The dotted line represents the value of $z$ at $T=0$ in [12]. The value of $z$ at finite temperatures converges to the value of about 0.45 to 0.5 in the dilute limit.

it tends to converge to approximately $z=0.45-0.5$ at finite temperatures.

The dynamic exponent $z$ accurately characterized the evolution of the average cluster size, as shown above. In the IDP system, however, charged and neutral clusters may grow differently and such hetero-aggregation dynamics cannot be understood simply from the analysis of the dynamic exponent. To determine the behaviors of charged and neutral clusters, the relative number of neutral to charged clusters,

$$
N_{\mathrm{c}}^{\mathrm{rel}}=\frac{N_{\mathrm{c}}^{0}}{N_{\mathrm{c}}^{+}+N_{\mathrm{c}}^{-}},
$$

is measured, where $N_{\mathrm{c}}^{+}+N_{\mathrm{c}}^{-}$and $N_{\mathrm{c}}^{0}$ are the numbers of charged and neutral clusters, respectively. The charge of a cluster is defined as the total dipole intensity in the cluster. Clusters are, therefore, classified into the following three types. For a cluster with size $s$,

$$
\sum_{i=1}^{s} \mu_{i}= \begin{cases}\mu_{0} & \text { positively charged cluster } \\ 0 & \text { neutral cluster, } \\ -\mu_{0} & \text { negatively charged cluster }\end{cases}
$$

Few clusters with a charge of more than $2 \mu_{0}$ are observed in the simulation, and therefore, a charged cluster consists of an odd number of particles and a neutral cluster has an even number of particles.

The time evolution of the relative cluster number is shown in Fig. 6. Although the exponent $z$ depends very weakly on $T(z \simeq 0.5)$, the dynamics clearly changes between $T=0.03$ and $T=0.04$. While $N_{\mathrm{c}}^{\mathrm{rel}}$ converges to a constant value around 0.4 at higher temperatures, it is likely to vanish as time becomes large at the lower temperatures. In the latter case $(T \leq 0.3)$, it appears that the hetero-aggregation effect remains.

It has already been reported that discrimination between charged and neutral clusters occurs in hetero aggregation for relatively small clusters (for example, clusters with fewer

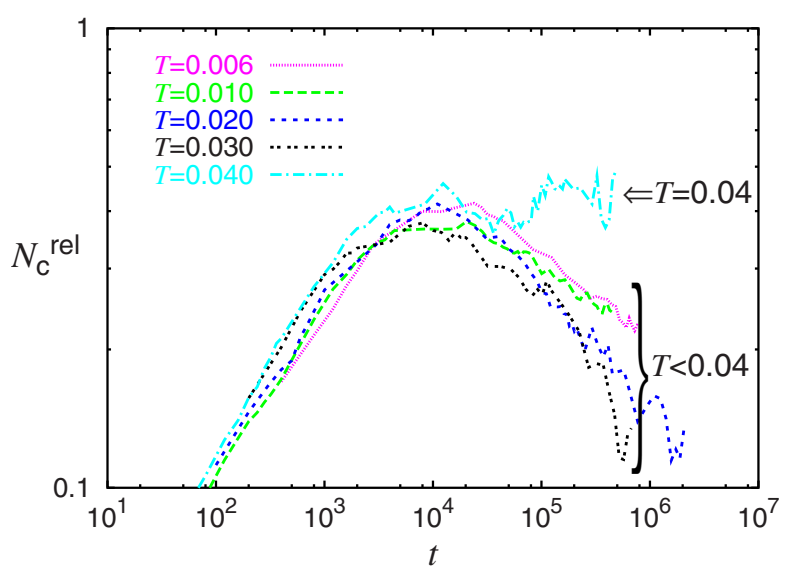

FIG. 6. (Color online) The time evolution of the relative cluster number $N_{\mathrm{c}}^{\mathrm{rel}}$ for $\phi=0.010$. Decimal logarithms are taken for both axes. The curves at $T \leq 0.03$ tend to vanish, while that at $T=0.04$ converges to a constant value.

than ten particles) $[7,12]$; however, in the present study, it is shown that such discrimination occurs even at a much later stage of aggregation.

\section{ANALYSIS}

\section{A. Structures}

The structures of IDP clusters are illustrated in Fig. 7. Although chainlike structures $(D \simeq 1.0)$ grow at very low temperatures, once microcrystalline structures with a square lattice appear at the temperature of around $T=0.002-0.006$, each cluster begins to form a fractal structure with $D$ $\simeq 1.45$.

To understand how thermal noise destroys a chainlike cluster and causes the formation of a more compact configuration, the analysis by Varga and Kun [14] is very useful. According to their analysis, when a chain with $N$ particles is bent with bending angle $\theta$, its energy is

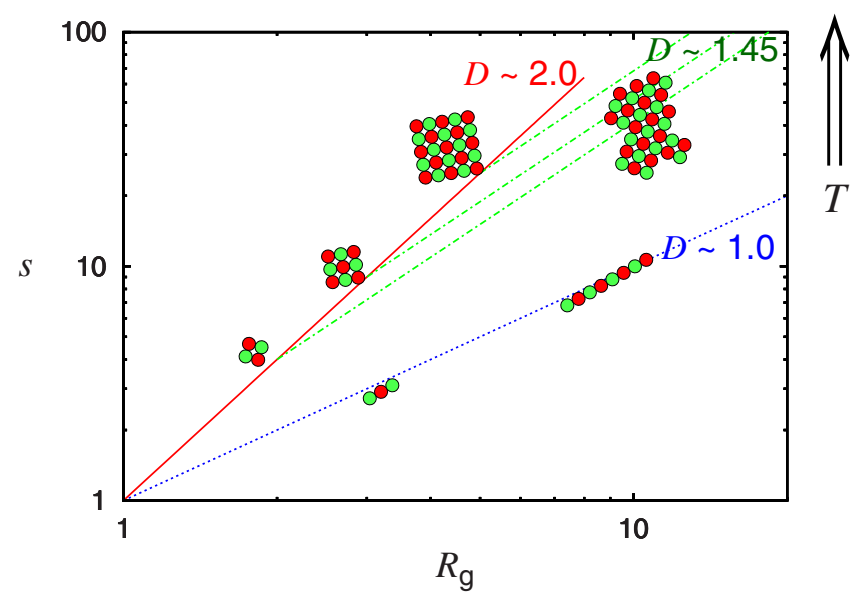

FIG. 7. (Color online) A schematic diagram of the fractal structures of IDP corresponding to Fig. 2. Chainlike clusters $(D=1.0)$ appear at low temperatures, while a crossover phenomenon from $D=2$ to $D=1.45$ is observed at higher temperatures. 


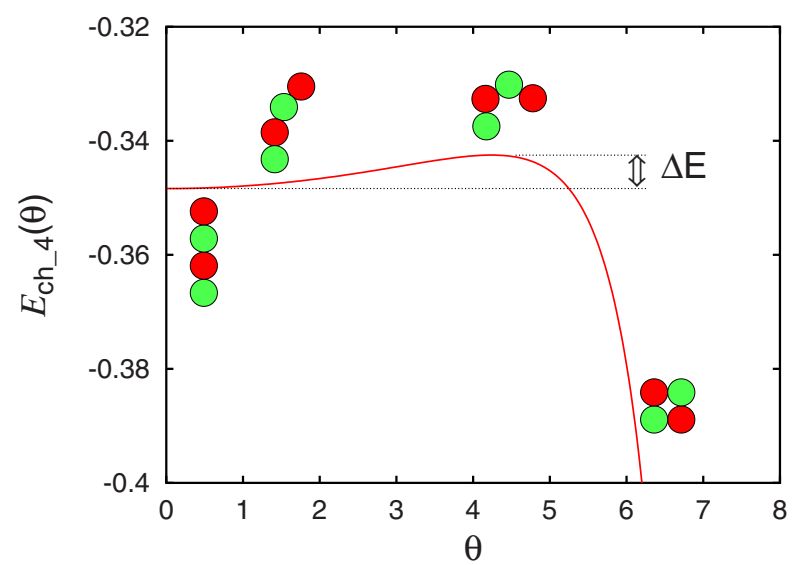

FIG. 8. (Color online) The energy of a four-particle chain as a function of bending angle $\theta$. There is a small energy barrier to the formation of the tetragonal configuration.

$$
E_{\text {ch_N } N}(\theta)=\frac{\mu_{0}^{2} \sin ^{3}(\theta / 2 N)}{(2 R)^{3}}\left[\sum_{k=1}^{N-1}(-1)^{k} \frac{N-k}{\sin ^{3}(k \theta / 2 N)}\right] .
$$

Figure 8 shows the curve of $E_{\text {ch_4 }}(\theta)$. The straight chain alignment $(\theta=0)$ is metastable and the ground-state configuration is tetragonal $(\theta=2 \pi)$. Between them, there is an energy barrier to the bending of the straight chain. The value of the energy barrier is $\Delta E=0.00587$. Thus, straight chains begin to fold by thermal motion at a temperature higher than 0.006 . This is consistent with the simulation result.

\section{B. Dynamic exponent}

The dynamic exponent $z$ estimated in the simulation was $z=0.45-0.5$ in the dilute limit. The theoretical value of the dynamic exponent for DLCA is compared with our result. Under Langevin noise, the amplitude of the diffusive motion of a cluster with size $s$ can be estimated as

$$
\left(\frac{\sum_{i=1}^{s} v_{i}}{s}\right)^{2} \simeq \frac{1}{s^{2}} \sum_{i=1}^{s} v_{i}^{2} \simeq \frac{s k_{\mathrm{B}} T}{m s^{2}} \propto s^{-1}
$$

thus, $\gamma=-1$ is substituted into Eq. (1). Then $z=0.5$ is obtained, which agrees well with our result. Thus the CCA dynamics at finite temperatures $(0.0002 \leq T)$ can be assumed to be that of normal DLCA from the viewpoint of the dynamic exponent.

\section{Smoluchowski equation analysis}

The characteristics of hetero-aggregation dynamics is discussed here, focusing on the behavior of $N_{\mathrm{c}}^{\mathrm{rel}}$. The curves for $T<0.04$ in Fig. 6 have a similar profile and they are likely to be fitted by a single curve upon suitably rescaling the horizontal axis. Taking into consideration the fact that the rate of growth of clusters at different temperatures is almost identical $\left(\bar{s} \sim t^{z}\right)$ and that the prefactor of $t^{z}$ depends on the temperature (see Fig. 4), $N_{\mathrm{c}}^{\text {rel }}$ is replotted against $\bar{s}$ instead of $t$ in

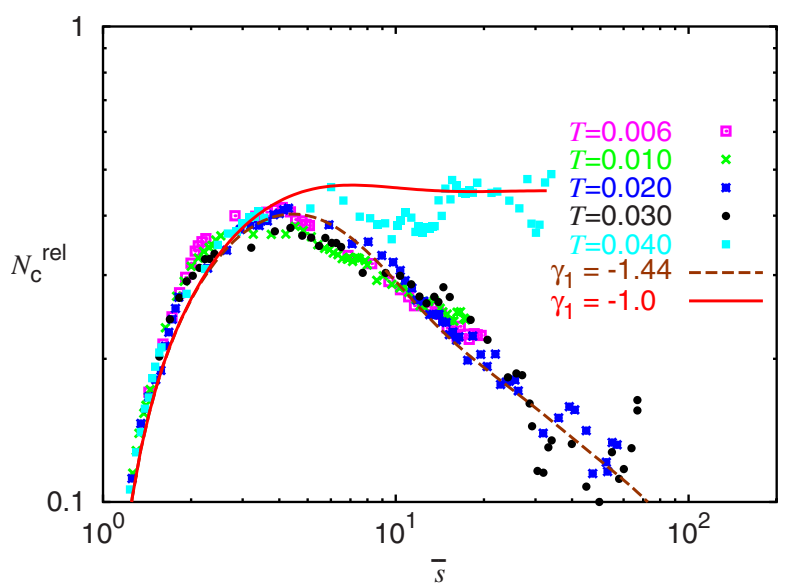

FIG. 9. (Color online) The time evolution of the relative number of neutral to charged clusters $N_{\mathrm{c}}^{\mathrm{rel}}$ against the average cluster size $\bar{s}$. Plots represent the simulation result and curves represent analytical values of the hetero-DLCA Smoluchowski equation for $\left(\gamma_{0}, \gamma_{1}\right)=$ $(-1,-1)$ and $\left(\gamma_{0}, \gamma_{1}\right)=(-1,-1.44)$.

Fig. 9. This time, the profiles of $N_{\mathrm{c}}^{\mathrm{rel}}(\bar{s})$ for $T<0.04$ almost lie on a single curve.

To theoretically investigate this behavior of $N_{\mathrm{c}}^{\text {rel }}$, a rate equation analysis is performed. Puertas et al. [7] introduced a set of equations generalizing the Smoluchowski equation to apply it to hetero aggregation; its form is

$$
\begin{gathered}
\frac{d}{d t} n_{s}^{+}=\sum_{i+j=s} K_{i j}^{0} n_{i}^{+} n_{j}^{0}-n_{s}^{+} \sum_{k=1}^{\infty}\left(K_{s k}^{1} n_{k}^{-}+K_{s k}^{0} n_{k}^{0}\right) \\
\frac{d}{d t} n_{s}^{-}=\sum_{i+j=s} K_{i j}^{0} n_{i}^{-} n_{j}^{0}-n_{s}^{-} \sum_{k=1}^{\infty}\left(K_{s k}^{1} n_{k}^{+}+K_{s k}^{0} n_{k}^{0}\right) \\
\frac{d}{d t} n_{s}^{0}=\sum_{i+j=s}\left(\frac{1}{2} K_{i j}^{0} n_{i}^{0} n_{j}^{0}+K_{i j}^{1} n_{i}^{+} n_{j}^{-}\right)-n_{s}^{0} \sum_{k=1}^{\infty} K_{s k}^{0}\left(n_{k}^{+}+n_{k}^{0}+n_{k}^{-}\right),
\end{gathered}
$$

where $n_{i}^{+}, n_{i}^{-}$, and $n_{i}^{0}$ are the densities of positive, negative, and neutral clusters with size $i$, respectively. The kernels $K_{i j}^{0}$ and $K_{i j}^{1}$ indicate the collision rates between clusters of size $i$ and size $j$. Equation (15) describes the following situation: (i) a pair of oppositely charged clusters of sizes $i$ and $j$ merges through $K^{1}$ to form a neutral cluster with of $i+j$, (ii) pairs of charged-neutral and neutral-neutral clusters merge through $K^{0}$ to form larger charged and neutral clusters, respectively, (iii) clusters with like charges do not merge with each other.

Puertas et al. [7] employed kernels with identical exponents of the form

$$
\begin{gathered}
K_{i j}^{0}=k_{0}(i+j)^{\lambda}, \\
K_{i j}^{2}=k_{1}(i+j)^{\lambda} .
\end{gathered}
$$

By changing the ratio between the constant prefactors $k_{0}$ and $k_{1}$, the segregation of dynamics between relatively small odd and even clusters is reproduced. However, we found that the 
TABLE I. A summary of the structures and dynamics of the IDP aggregation process.

\begin{tabular}{lccc}
\hline \hline & Structures & Dynamic exponent & Hetero-aggregation dynamics \\
\hline$T<0.006$ & $\begin{array}{c}\text { Straight chains } \\
(D \simeq 1.0)\end{array}$ & $z \simeq 0.2$ & Hetero-ALCA \\
$0.006 \leq T<0.04$ & $\begin{array}{c}\text { Crossover from } D \simeq 2.0 \\
\text { to } D \simeq 1.45 .\end{array}$ & $z \simeq 0.5$ & $\begin{array}{c}\text { Diffusive aggregation with weak attraction } \\
\text { between charged-charged cluster pairs } \\
\left.\text { (Smoluchowski eq.: } \gamma_{0}=-1.0, \gamma_{1}=-1.45\right) \\
0.04 \leq T\end{array}$ \\
& $\begin{array}{c}\text { The crossover size increases with } T . \\
\text { Normal square lattice }(D \simeq 2.0)\end{array}$ & $z \simeq 0.5$ & $\begin{array}{l}\text { Normal DLCA } \\
\left(\gamma_{0}=\gamma_{1}=-1.0\right)\end{array}$ \\
\hline \hline
\end{tabular}

segregating phenomenon for a long time cannot be represented with those kernels and that $N_{\mathrm{c}}^{\mathrm{rel}}$ monotonically converge into a constant value.

Since it is known that the dynamics of normal DLCA is well described by the original Smoluchowski equation using a single kernel [19]

$$
K_{i j}=\left(i^{\gamma}+j^{\gamma}\right),
$$

we employ kernels with the form

$$
\begin{aligned}
& K_{i j}^{0}=\left(i^{\gamma_{0}}+j^{\gamma_{0}}\right), \\
& K_{i j}^{1}=\left(i^{\gamma_{1}}+j^{\gamma_{1}}\right) .
\end{aligned}
$$

The exponents $\gamma_{0}$ and $\gamma_{1}$ are used as control parameters.

The time evolution of Eq. (15) is calculated numerically from the initial conditions

$$
\begin{gathered}
n_{1}^{+}=n_{1}^{-}=0.5, \\
n_{i}^{+}=n_{i}^{-}=0 \quad(i \neq 1), \\
n_{i}^{0}=0 \quad(\text { for all } i) .
\end{gathered}
$$

First, we take the values

$$
\gamma_{0}=\gamma_{1}=-1,
$$

since the exponent for the normal DLCA is $\gamma=-1$. The time evolution of $N_{\mathrm{c}}^{\mathrm{rel}}$ for the values given in Eq. (20) is shown in Fig. 9 and is indicated as $\gamma_{1}=-1.0$. The curve agrees well with the simulation result at $T=0.04$. Therefore, the CCA dynamics at temperatures higher than 0.04 can be regarded as that of normal DLCA.

Next, the situation for $0.006 \leq T<0.04$ is considered. It is assumed that, in this temperature range, neutral clusters move purely by thermal diffusion, and that weak attractive interaction remains only between charged-charged clusters. To describe this situation, the value of $\gamma_{0}$ is fixed to -1 and $\gamma_{1}$ is varied as the fitting parameter. As shown in Fig. 9, the analytical curve closely reproduces the simulation result when the exponents are set to

$$
\gamma_{0}=-1, \quad \gamma_{1}=-1.44
$$

Thus, it can be concluded that the hetero-aggregation dynamics in the range of $0.006 \leq T \leq 0.03$ is caused by the diffusive motion of each cluster and the weak attraction between oppositely charged cluster pairs.

\section{SUMMARY}

The cluster-cluster aggregation processes of Ising dipolar particles under thermal noise were investigated numerically and theoretically. As the temperature increases, the typical cluster structure changes from chainlike configurations $(D$ $\simeq 1)$ to fractal clusters $(D \simeq 1.45)$ consisting of small crystalline domains (with $D \simeq 2.0$ ) at approximately $T=0.006$. The crossover size from $D=2.0$ to $D=1.45$ increases as the temperature increases and diverges at $T=0.04$. Therefore, all clusters form the crystalline structure at a temperature higher than $T=0.04$. The transition from chains to fractal clusters can be well explained theoretically by considering the energy barrier to a chain being bent into a tetragonal configuration. Once the thermal motion overcomes the energy barrier, microcrystalline clusters are constructed at the smallest scale, then these clusters grow by merging with each other to form fractal structures.

The dynamic exponent for the average cluster size $z$ is estimated to be approximately 0.5 at finite temperatures, which differs from the value of about 0.2 at $T=0$. The value of 0.5 is identical with the theoretical value for normal DLCA. Although it was not shown in the previous section, we also observed another dynamic exponent $z^{\prime}$, which corresponds to the time evolution of the total cluster number $N_{\mathrm{c}}$ as $N_{\mathrm{c}}(t) \sim t^{-z^{\prime}}$. The value of $z^{\prime}$ was also estimated to be approximately 0.5 at finite temperatures.

Within the temperature range where $z=0.5$ is observed, another change occurs in the relative dynamics between charged and neutral clusters. While the ratio of neutral clusters to charged clusters $N_{\mathrm{c}}^{\mathrm{rel}}$ converge into a constant value at $0.04 \leq T$, it tends to vanish at lower temperatures as the longtime behavior. This vanishing behavior of $N_{\mathrm{c}}^{\mathrm{rel}}(t)$ was convincingly explained on the basis of mean field analysis. Using the Smoluchowski eq. with a twofold kernel, the curve of $N_{\mathrm{c}}^{\mathrm{rel}}$ is closely reproduced by setting the kernel for charged cluster pairs to have $\gamma_{1}=-1.44$, which suggests that the motion of clusters is nearly diffusive but there is weak attraction among charged clusters. On the other hand, the time evolution of $N_{\mathrm{c}}^{\mathrm{rel}}$ at $0.04 \leq T$ is closely reproduced by the Smoluchowski equation using the kernels for normal diffusion $\left(\gamma_{0}=\gamma_{1}=-1\right)$.

The structures and dynamics of the aggregation process are summarized in the Table I. As it shows, the transitions of structures and changes in aggregation dynamics occur at approximately the same time. 


\section{DISCUSSION}

As mentioned above, the changes in the cluster structure involve a change in aggregation dynamics. This finding is to be expected, because the intensity of attraction between charged clusters depends on the cluster shape. For example, while the attractive field near each end of a chain is strong and its intensity is almost the same as that of an isolated $\mu_{0}$ or $-\mu_{1}$ particle, the attraction of a cluster with a more compact structure becomes relatively weak because the charge of a particle is shielded by the opposite charges of neighboring particles. In particular, the charge in a tetragonal crystalline cluster is almost negligible, and such a cluster moves purely as a result of the thermal noise, as our results show.

As described in the previous section, the long-time evolution of the relative cluster number $N_{\mathrm{c}}^{\text {rel }}$ cannot be explained by the kernels in Eq. (16) which use identical exponents for charged and neutral clusters, but it is well explained by the kernels with different exponents given in Eq. (18). This difference in exponents corresponds to the different motion of charged and neutral clusters. The fact that the exponent of the kernel for charged-charged cluster pairs differs from the value for normal diffusion $\left(\gamma_{1} \neq-1\right)$ is clearly due to the attractive force between these clusters. The origin of the value $\gamma_{1}=-1.44$ will be clarified in further studies.

It is also worth noting that the dynamic exponent $z$ has the same value 0.5 even if $\gamma_{1}$ changes from -1 to -1.44 . Additionally, $\gamma_{0}$ is fixed at -1 for both cases. This suggests that only the exponent $\gamma_{0}$ affects the rate of growth of the average cluster size regardless of $\gamma_{1}$.
The behavior of $N_{\mathrm{c}}^{\mathrm{rel}}$ in the temperature region $T \leq 0.002$ was not discussed in previous sections. We observed in the simulation that $N_{\mathrm{c}}^{\mathrm{rel}}(t)$ also decreases as with time; however, the profile is slightly different from that in $0.006 \leq T<0.04$. Although this behavior was not reproduced using Eq. (15) with the set of kernels in Eq. (18), it is expected to be understood by analysis using the Smoluchowski equation with a different set of kernels. The reason why the dynamic exponent $z$ in the low-temperature region (and at $T=0$ ) is 0.2 , is also not yet revealed. At such a low temperature, there may even be attraction between a neutral-neutral cluster pair. Further investigation is needed to understand the hetero-ALCA dynamics at extremely low temperatures. Investigation of the dynamics at extremely low temperatures is important since it corresponds to the environment of experiments in $[16,7]$, in which the motion of dipolar particles is highly dissipative and thermal noise is negligible.

\section{ACKNOWLEDGMENTS}

The authors thank T. Shimada and H. Watanabe for fruitful discussions. The present study was supported by the 21 st COE program, "Frontiers of Computational Science," Nagoya University, and by Grants-in-Aid for Scientific Research (Contract No. 19340110) from the Ministry of Education, Culture, Sports, Science and Technology, and by KAUST GRP(KUK-I1-005-04). F.K. was supported by the Hungarian Academy of Sciences.
[1] T. Vicsek, Fractal Growth Phenomena (World Scientific, Singapore, 1989).

[2] P. Meakin, J. Sol-Gel Sci. Technol. 15, 97 (1999).

[3] T. Vicsek and F. Family, Phys. Rev. Lett. 52, 1669 (1984).

[4] P. Meakin, T. Vicsek, and F. Family, Phys. Rev. B 31, 564 (1985).

[5] M. Kolb, Phys. Rev. Lett. 53, 1653 (1984).

[6] J. M. López-López, A. Schmitt, J. Callejas-Fernández, and R. Hidalgo-Álvarez, Phys. Rev. E 69, 011404 (2004).

[7] A. M. Puertas, A. Fernández-Barbero, and F. J. de las Nieves, Physica A 304, 340 (2002).

[8] H. See and M. Doi, J. Phys. Soc. Jpn. 60, 2778 (1991).

[9] G. Helgesen, A. T. Skjeltorp, P. M. Mors, R. Botet, and R. Jullien, Phys. Rev. Lett. 61, 1736 (1988).

[10] H. Morimoto and T. Maekawa, J. Phys. A 33, 247 (2000).

[11] W. Wen, F. Kun, K. F. Pál, D. W. Zheng, and K. N. Tu, Phys.
Rev. E 59, R4758 (1999).

[12] N. Yoshioka, I. Varga, F. Kun, S. Yukawa, and N. Ito, Phys. Rev. E 72, 061403 (2005).

[13] I. Varga, F. Kun, and K. F. Pál, Phys. Rev. E 69, 030501(R) (2004).

[14] I. Varga and F. Kun, Philos. Mag. 86, 2011 (2006).

[15] I. Varga, N. Yoshioka, F. Kun, and S. Gang, J. Stat. Mech. 2007, P09015.

[16] W. D. Ristenpart, I. A. Aksay, and D. A. Saville, Phys. Rev. Lett. 90, 128303 (2003).

[17] I. Varga, H. Yamada, F. Kun, H.-G. Matuttis, and N. Ito, Phys. Rev. E 71, 051405 (2005).

[18] M. Suzuki, F. Kun, S. Yukawa, and N. Ito, Phys. Rev. E 76, 051116 (2007).

[19] K. Kang, S. Redner, P. Meakin, and F. Leyvraz, Phys. Rev. A 33, 1171 (1986). 